Departamento de Neurología, Clínica Las Condes. Santiago, Chile.

Los autores declaran no tener conflictos de interés. Trabajo no recibió financiamiento.

Recibido el 17 de agosto de 2017, aceptado el 18 de enero de 2018.

Correspondencia a:

Dr. Marcelo Miranda Lo Fuentecilla 441. Santiago, Chile. marcelomirandac@gmail.com

\section{Severo edema inducido por la terapia dopaminérgica en la enfermedad de Parkinson. Caso clínico}

\author{
MARCELO MIRANDA C., LORENA HUDSON A.
}

\section{Severe leg edema associated with the use of dopaminergic drugs in Parkinson's disease. Report of one case}

The main adverse effects of dopaminergic drugs used in Parkinson's disease are hypotension, somnolence, hallucinations and impulse control disorder. Less common is leg edema. We report on a 68-year-old male receiving levodopa and pramipexole consulting for severe leg edema lasting two years, whose etiology was not ascertained with multiple lab tests. This edema subsided substantially when pramipexole was discontinued and the dose of levodopa was increased to treat motor symptoms.

(Rev Med Chile 1624-1625)

Key words: Dopamine Agents; Drug-Related Side Effects and Adverse Reactions; Edema; Parkinson Disease.
L

a enfermedad de Parkinson es una enfermedad neurodegenerativa básicamente del sistema nervioso central, cuya terapia sintomática es en base a fármacos dopaminérgicos como levodopa y agonistas de la dopamina.

El propósito de esta comunicación es alertar de una reacción adversa de los fármacos usados en la terapia de esta condición, en particular los agonistas dopaminérgicos que están en el programa de garantía explícitas de salud (AUGE).

Estos fármacos frecuentemente ocasionan efectos secundarios como hipotensión, hipersomnolencia, descontrol de impulsos, alucinaciones, etc. ${ }^{1,2}$.

La presencia de edema de extremidades inferiores asociado al uso de pramipexole y ropinerole es un hecho bien reconocido en la literatura, pero poco conocido en nuestro medio en cuanto a la magnitud e incapacidad que puede alcanzar. Por este motivo comunicamos un paciente que presentó esta reacción adversa reversible con la suspensión del fármaco.

\section{Caso clínico}

Varón de 68 años con 10 años de historia de enfermedad de Parkinson tratado con levodopa $600 \mathrm{mg}$ día y pramipexole $3 \mathrm{mg}$ día y sin patología cardiovascular de base. Acudió a consulta por intenso edema que presentaba hacía 2 años en extremidades inferiores y que le dificultaba marcadamente la deambulación. Había sido sometido a un extenso estudio cardiovascular y vascular periférico, sin determinar origen del problema y el uso de diuréticos y drenaje linfático no redujo el edema.

En el examen general, aparte de su signología parkinsoniana, destacaba un gran edema de extremidades inferiores hasta los muslos, no inflamatorio, blando y sin patología venosa visible, ni alteración cutánea (Figura 1). El edema impedía el uso de calzado normal y dificultaba notoriamente la marcha al enlentecerla aun más.

Se decidió la suspensión de pramipexole, con el ajuste compensatorio para sus síntomas moto- 

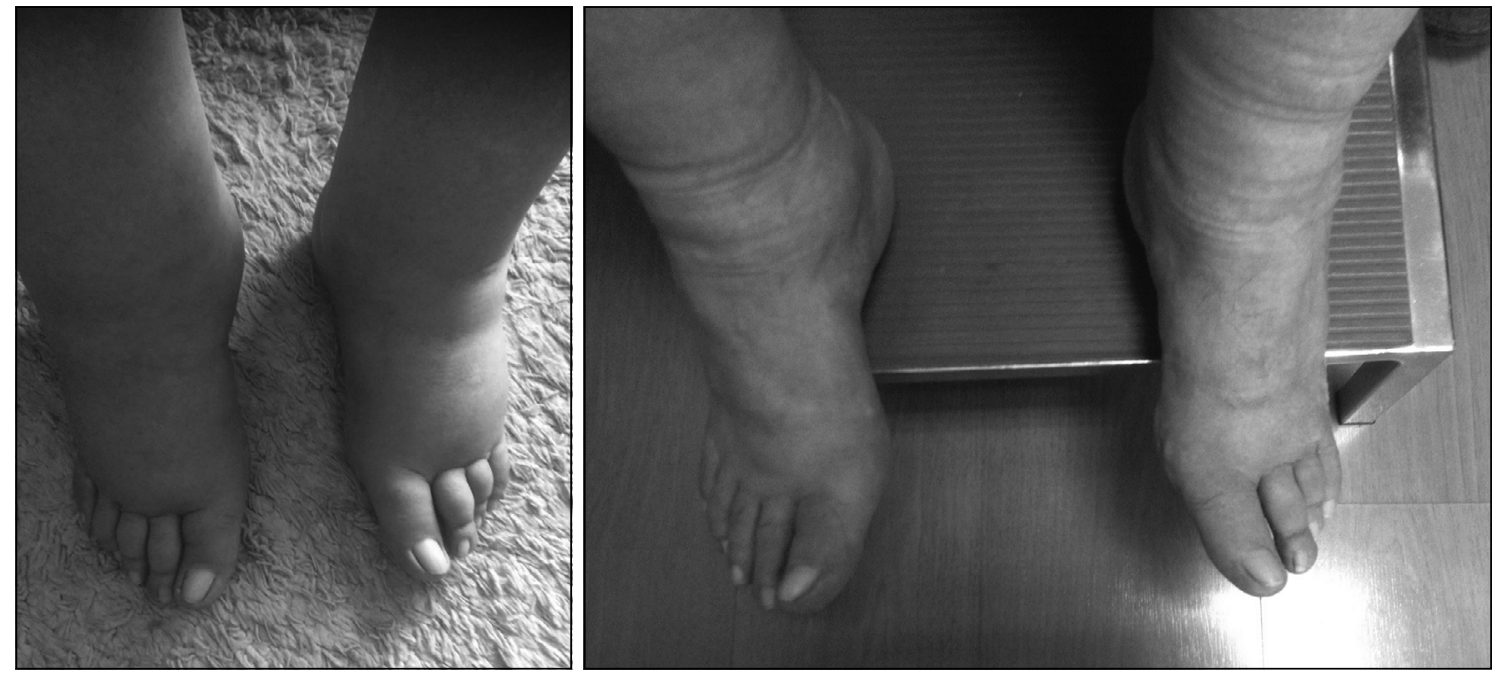

Figura 1 A y B. Edema de extremidades previo y posterior a la suspensión del fármaco.

res con mayor dosis de levodopa, lo que generó una marcada reducción del edema en 2 meses (Figura 1 ).

\section{Comentario}

Esta reacción adversa idiosincrática no es infrecuente de observar en pacientes tratados con estos fármacos, se presenta entre $5 \%$ y $20 \%{ }^{3,4}$ de los pacientes, aun con dosis bajas de estos fármacos y la conducta es el retiro del medicamento. Esta reacción adversa también ha sido descrita con el uso de agonistas de la dopamina en el síndrome de piernas inquietas y también con otros fármacos no dopaminérgicos como amantadina ${ }^{3,5}$.

No está aclarada la fisiopatología de este efecto secundario, sin embargo, como la dopamina es un regulador del tono del sistema simpático, de la secreción de aldosterona y tiene efectos en canales de $\mathrm{Na}-\mathrm{K}+$ mediados por adenosina trifosfato, el efecto de los agonistas a estos niveles puede influir en su patogenia ${ }^{4}$. Se han descrito algunos factores de riesgo, siendo el más importante la cardiopatía coronaria ${ }^{4,6}$.

Creemos importante dar a conocer esta reacción adversa que no es muy conocida en nuestro medio, para evitar someter a los pacientes a estudios injustificados, además que es reversible con la conducta adecuada.

\section{Referencias}

1. Biglan KM, Holloway RG, McDermott MP. Risk factors for somnolence, edema, and hallucinations in early Parkinson disease. Neurology 2007; 69: 187-95.

2. Miranda M, Slachevsky A, Bustamante L, Pérez C. Juego patológico como efecto secundario del tratamiento de la enfermedad de Parkinson. Rev Med Chile 2010; 138: 521-2.

3. Tan EK, Ondo W. Clinical characteristics of pramipexole-induced peripheral edema. Arch Neurol 2000; 57: 729-32.

4. Cicero CE, Nicolita A, Motile G, Zappia M. A case of severe leg edema in a patient with Parkinson's disease treated with pramipexole. Postgrad Med J 2016; 92: 484.

5. British National Formulary. Editado por The Royal Pharmaceutical Society, Pharmaceutical Press. Londres. 2017; 382-400.

6. Zavala J, Muñoz R, Teide H. Pramipexole-related chronic lower limb edema in a patient with Parkinson's disease. J Clin Neurosci 2012;19: 1298-9. 